\title{
Relaxation in hypertrophic cardiomyopathy and hypertensive heart disease: relations between hypertrophy and diastolic function
}

\author{
S F De Marchi, Y Allemann, C Seiler
}

\begin{abstract}
Aim-To determine the relation between the extent and distribution of left ventricular hypertrophy and the degree of disturbance of regional relaxation and global left ventricular filling. Methods-Regional wall thickness (rWT) was measured in eight myocardial regions in 17 patients with hypertrophic cardiomyopathy, 12 patients with hypertensive heart disease, and 10 age matched normal subjects, and an asymmetry index calculated. Regional relaxation was assessed in these eight regions using regional isovolumetric relaxation time (rIVRT) and early to late peak filling velocity ratio ( $\mathrm{rE} / \mathrm{A}$ ) derived from Doppler tissue imaging. Asynchrony of rIVRT was calculated. Doppler left ventricular filling indices were assessed using the isovolumetric relaxation time, the deceleration time of early diastolic filling (E-DT), and the E/A ratio.

Results-There was a correlation between rWT and both rIVRT and rE/A in the two types of heart disease (hypertrophic cardiomyopathy: $r=0.47, \mathrm{p}<0.0001$ for rIVRT; $r=-0.20, \mathrm{p}<0.05$ for $\mathrm{rE} / \mathrm{A}$; hypertensive heart disease: $r=0.21, \mathrm{p}<0.05$ for $\mathrm{rIVRT} ; r=-0.30, \mathrm{p}=0.003$ for $\mathrm{rE} / \mathrm{A})$. The degree of left ventricular asymmetry was related to prolonged E-DT $(r=0.50$, $\mathrm{p}=0.001)$ and increased asynchrony $(r=0.42, \mathrm{p}=0.002)$ in all patients combined, but not within individual groups. Asynchrony itself was associated with decreased E/A $(r=-0.39$, $\mathrm{p}=0.01)$ and protracted E-DT $(r=0.69, \mathrm{p}<0.0001)$ and isovolumetric relaxation time $(r=0.51, \mathrm{p}=0.001)$ in all patients. These correlations were still significant for E-DT in hypertrophic cardiomyopathy $(r=0.56, \mathrm{p}=0.02)$ and hypertensive heart disease $(r=0.59, \mathrm{p}<0.05)$ and for isovolumetric relaxation time in non-obstructive hypertrophic cardiomyopathy $(\mathrm{n}=8$, $r=0.87, \mathrm{p}=0.005)$.

Conclusions-Non-invasive ultrasonographic examination of the left ventricle shows that in both hypertrophic cardiomyopathy and hypertensive heart disease, the local extent of left ventricular hypertrophy is associated with regional left ventricular relaxation abnormalities. Asymmetrical distribution of left ventricular hypertrophy is indirectly related to global left ventricular early filling abnormalities through regional asynchrony of left ventricular relaxation. (Heart 2000;83:678-684)
\end{abstract}

Keywords: hypertrophic cardiomyopathy; hypertensive heart disease; isovolumetric relaxation; diastolic function

Hypertrophic cardiomyopathy is a primary myocardial disease. It is characterised by left ventricular hypertrophy which develops independently of loading conditions. Hypertrophy in hypertensive heart disease is considered to be a response to increased afterload. ${ }^{1}$ In both types of hypertrophy, abnormal left ventricular relaxation is a hallmark of the disease. Even in the presence of normal left ventricular systolic performance, this abnormality results in a rise in left atrial pressure, pulmonary congestion, and oedema. Dyspnoea is thus a very common symptom in these patients. ${ }^{23}$

Myocardial hypertrophy has been shown to be the crucial morphological change accounting for abnormal relaxation, ${ }^{4-9}$ whereas increased interstitial fibrosis appears to have little impact on relaxation, but primarily affects diastolic myocardial stiffness. ${ }^{6}{ }^{11}$ The histological hallmark of hypertrophic cardiomyopathy - myocardial fibre disarray - may affect both ventricular relaxation and stiffness. Relaxation properties of the myocardium, however, can vary considerably between different regions. This temporal and spatial nonuniformity is an important determinant of global left ventricular relaxation in coronary and hypertensive heart disease and hypertrophic cardiomyopathy. ${ }^{92-16}$ Regional relaxation abnormalities may precede and finally cause global ventricular diastolic dysfunction. So far, the relation between the pattern of left ventricular hypertrophy and regional left ventricular relaxation abnormalities and the global left ventricular filling pattern has not been investigated comprehensively.

Doppler tissue imaging allows non-invasive assessment of regional left ventricular myocardial long axis function by measuring basoapically directed wall motion velocities. ${ }^{17}{ }^{18}$ From the time course of contractionrelaxation motion in different myocardial segments, regional myocardial relaxation indices and the synchronisation of particular events in the cardiac cycle can be derived. ${ }^{19-22}$

The purpose of this non-invasive study in patients with primary and secondary left ventricular hypertrophy was to determine the relation between the extent and distribution of left ventricular hypertrophy on the one hand, and the degree of disturbed regional relaxation and global left ventricular filling on the other (table 1). 
Table 1 Description of hypertrophy and relaxation indices

\begin{tabular}{|c|c|}
\hline Index & Description \\
\hline \multicolumn{2}{|l|}{ Left ventricular hypertrophy } \\
\hline Regional wall thickness & $\begin{array}{l}\text { Wall thickness of each of the eight segments } \\
\text { measured using cross sectional echocardiography }\end{array}$ \\
\hline Global left ventricular hypertrophy & Sum of all regional wall thickness measurements \\
\hline Asymmetry index & $\begin{array}{l}\text { Coefficient of variation of all regional wall thickness } \\
\text { measurements }\end{array}$ \\
\hline \multicolumn{2}{|l|}{ Diastolic left ventricular function } \\
\hline Regional relaxation indices & $\begin{array}{l}\text { DTI derived IVRT and E/A in each wall segment } \\
(\text { rIVRT, rE/A) }\end{array}$ \\
\hline $\begin{array}{l}\text { Global left ventricular relaxation } \\
\text { indices }\end{array}$ & $\begin{array}{l}\text { Doppler derived IVRT, E/A, and E-DT of } \\
\text { transmitral inflow }\end{array}$ \\
\hline Asynchrony index & $\begin{array}{l}\text { Coefficient of variation of R-rIVRT time of all wall } \\
\text { segments }\end{array}$ \\
\hline
\end{tabular}

$\overline{\text { DTI, Doppler tissue imaging; E/A, early to late diastolic peak velocity; E-DT, deceleration time of }}$ early diastolic filling; IVRT, isovolumetric relaxation time; R-rIVRT time, time from ECG derived

$\mathrm{R}$ wave to DTI derived beginning of isovolumetric relaxation.

\section{Methods}

PATIENTS

Thirty nine patients (mean (SD) age, 52 (17) years) were included in this prospective study. Patients with conduction abnormalities and pacemaker rhythm were excluded. Extrasystolic and postextrasystolic heart beats were not analysed.

Seventeen patients had hypertrophic cardiomyopathy, defined as left ventricular hypertrophy with an echocardiographically determined septal to posterior or anterior to inferior wall thickness ratio greater than 1.5. Absence of detectable causes for the development of left ventricular hypertrophy was mandatory for inclusion in this group.

Twelve patients with hypertensive heart disease were included. Inclusion criteria for this group were the presence of left ventricular hypertrophy and a history of systemic hypertension.

Ten subjects with normal echocardiography and with no history of cardiovascular disease were included as a control group.

All participants gave informed consent for their participation in the study.

ECHOCARDIOGRAPHY

All patients underwent transthoracic echocardiography in the left lateral supine position. The studies were performed using an Acuson Sequoia or Acuson XP128 Doppler ultrasonography system (Acuson Inc, Mountain View, California, USA). Both systems were equipped with $2.5-5.0 \mathrm{MHz}$ phased array cross sectional transducers, harmonic imaging, and Doppler tissue imaging systems.

Left ventricular hypertrophy, regional wall thickness, and hypertrophy distribution

Left ventricular mass index calculations using M mode (one dimensional) echocardiography, according to the conventions of the American Society of Echocardiography (ASE) for the assessment of the extent of left ventricular hypertrophy, ${ }^{23-25}$ are not accurate in the presence of asymmetrical hypertrophy. Therefore, a left ventricular wall thickness index was calculated. ${ }^{26}{ }^{27}$ The thickness of each wall region chosen for the assessment of regional wall motion velocities was measured from a cross sectional image in the parasternal short axis view. The left ventricular wall thickness index was calculated by adding the wall thickness measurements in each of the eight myocardial regions (table 1). This index is considered to be a quantitative reflection of the overall extent of left ventricular hypertrophy. ${ }^{26}$ In addition, the coefficient of variation of all regional wall thickness measurements within each left ventricle was calculated to quantify the degree of asymmetry of the hypertrophy (left ventricular asymmetry index), with low values reflecting symmetrical and high values reflecting asymmetrical ventricular geometry (table 1).

\section{Doppler tissue imaging of the left ventricle}

Doppler tissue imaging is a modification of the conventional Doppler flow imaging technology. Using filtering algorithms, tissue derived, slow motion Doppler signals $(\approx 10 \mathrm{~cm} / \mathrm{s})$ can be discriminated from blood flow Doppler signals, which are of much lower intensity in a comparable velocity range. Tissue derived Doppler signals can be displayed either as time-velocity tracings or as cross sectional or M mode colour images. ${ }^{17} 182829$ Pulsed wave Doppler tissue imaging was performed from the apical four and two chamber views. The sample volume was placed at eight regions of the left ventricle as follows: septal, lateral, inferior, and anterior left ventricular wall, with each wall segment at two different levels (midventricle and left ventricular base). Images of at least three cardiac cycles were obtained in each region. The regional isovolumetric relaxation time (rIVRT, ms) was defined as the time from the end of systolic contraction motion to the beginning of early diastolic expansion motion (E wave, fig 1). The early to late peak diastolic myocardial velocity ratio ( $\mathrm{rE} / \mathrm{A}$ ) as well as the time from the $\mathrm{R}$ wave on the ECG to the beginning of regional isovolumetric relaxation (R-rIVRT time, ms) were also measured for each region (table 1). In 150 patients at our laboratory with normal echocardiograms and left ventricular hypertrophy, interobserver and intraobserver variability (that is, the standard error of the estimate, SEE) of rIVRT was $21 \mathrm{~ms}$ and $17 \mathrm{~ms}$, respectively. Interobserver variability of rE/A was larger, which was mainly attributable to inaccuracies in rA measurements $(\mathrm{SEE}=0.32)$.

Global left ventricular filling indices

Left ventricular filling indices were obtained from pulsed wave Doppler recordings of the transmitral inflow pattern. The sample volume was placed at the tips of the mitral valve leaflets. Measurements included the early to late diastolic peak flow velocity ratio (E/A), the deceleration time of early transmitral filling (E-DT, ms), and the isovolumetric relaxation time (ms), which was defined as the time between the end of systolic left ventricular outflow and the beginning of transmitral inflow (table 1).

\section{Asynchrony of the beginning of regional} isovolumetric relaxation

In Doppler tissue imaging, the end of regional contraction motion and the onset of relaxation 


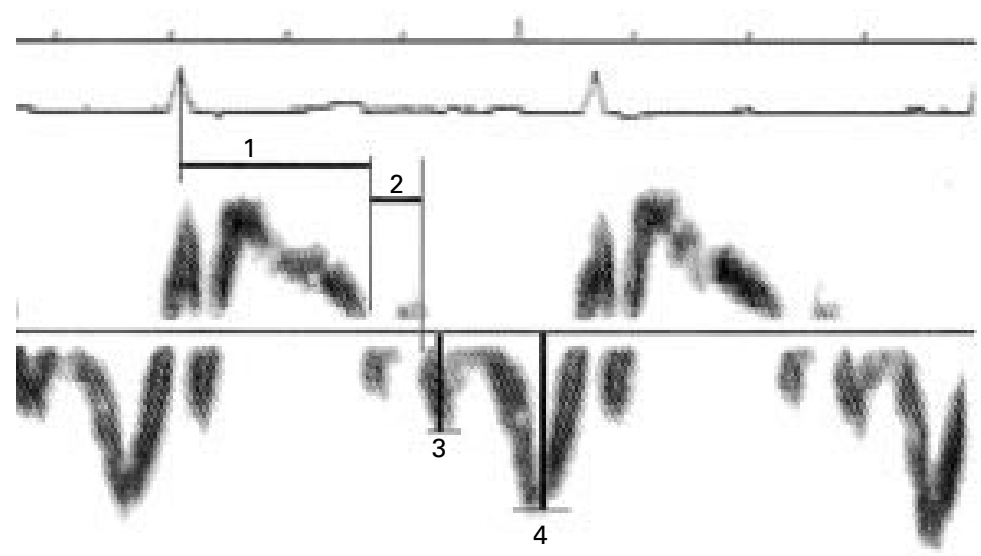

Figure 1 Example of a Doppler tissue velocity tracing at a septal basal myocardial region in a patient with hypertensive heart disease: (1), the time from the ECG $R$ wave to the end of contraction; (2), the regional isovolumetric relaxation time; (3), peak early diastolic motion velocity (E); (4), peak late diastolic motion velocity $(A)$.

Table 2 Patient characteristics

\begin{tabular}{llll}
\hline & $H C M(n=17)$ & $H T(n=12)$ & $N(n=10)$ \\
\hline General characteristics & $52(18)$ & $58(16)$ & $46(16)$ \\
Age & $13 / 4$ & $11 / 1$ & $8 / 2$ \\
Sex (male/female) & $63(9)$ & $74(11)^{\star}$ & $63(7)$ \\
Heart rate (beats/min) & $135(17)$ & $162(20)^{\star}$ & $124(11)$ \\
Systolic BP (mm Hg) & $75(9)$ & $92(14)^{\star}$ & $75(9)$ \\
Diastolic BP (mm Hg) & 5 & 3 & 0 \\
Vasoactive drugs & 0 & 2 & 0 \\
$\beta$ Blockers & $10^{\star}$ & 3 & 0 \\
Nitrates & 0 & 2 & 0 \\
Calcium antagonists & & & \\
ACE inhibitors & & & \\
\hline
\end{tabular}

Values are mean (SD) or $\mathrm{n}$.

${ }^{\star} \mathrm{p}<0.05 v$ the other two groups.

$\mathrm{ACE}$, angiotensin converting enzyme; BP, blood pressure; HCM, hypertrophic cardiomyopathy; HT, hypertensive heart disease; $\mathrm{N}$, normal controls.

can easily be determined (fig 1). The coefficient of variation of the time from the ECG R wave to this event of the cardiac cycle (R-rIVRT time) was calculated to determine the synchronicity of the beginning of regional isovolumetric relaxation (asynchrony index, table 1). A high index reflects a high intracardiac heterogeneity of the R-rIVRT time interval, and hence an asynchronous beginning of regional isovolumetric relaxation.

\section{STATISTICAL ANALYSIS}

Demographic, clinical, and echocardiographic data are expressed as mean (SD). For comparisons of continuous values between the study groups, analysis of variance (ANOVA) followed by Scheffé's test was performed. A $\chi^{2}$ test was used for comparison of categorical variables between the study groups. Relations between different echocardiographic variables were studied using linear regression analysis. A value of $\mathrm{p}<0.05$ was considered significant.

\section{Results}

PATIENT CHARACTERISTICS AND

ECHOCARDIOGRAPHIC DATA

Patient characteristics and echocardiographic data are listed in tables 2 and 3, respectively. Heart rate did not change significantly during the echocardiographic study in any of the groups. Interventricular septal thickness was greatest in hypertrophic cardiomyopathy, intermediate in hypertensive heart disease, and least in the normal subjects $(\mathrm{p}<0.05$ for all comparisons). Wall thickness index was significantly lower in the normal group than in the other two groups, whereas no significant difference was found between hypertensive heart disease and hypertrophic cardiomyopathy. The left ventricular ejection fraction was higher in the cardiomyopathy group than in the other two groups.

LEFT VENTRICULAR HYPERTROPHY AND DOPPLER INDICES OF REGIONAL RELAXATION

In hypertrophic cardiomyopathy, the coefficient of variation of wall thickness (asymmetry index) was significantly higher than in the other groups. There was an overall correlation between regional wall thickness and regional isovolumetric relaxation time (rIVRT), and an inverse overall correlation between wall thickness and regional $\mathrm{E} / \mathrm{A}$ ratio ( $\mathrm{rE} / \mathrm{A}$; table 4$)$. The correlations were significant for both hypertrophic cardiomyopathy and hypertensive heart disease, but not for the normal group. The relations between wall thickness and rIVRT were similar in hypertrophic cardiomyopathy and hypertensive heart disease (fig 2). In addition, regional relaxation indices were compared between wall segments without hypertrophy $(\leqslant 12 \mathrm{~mm})$ in the patients with hypertrophic cardiomyopathy and the corresponding segments in normal subjects. In these segments, regional isovolumetric relaxation time was greater and regional E/A ratio lower in the cardiomyopathy group than in the normal group ( $p<0.0001$ for both comparisons).

Although there was a significant overall correlation between the asymmetry index and the asynchrony index in all the subjects grouped together, no such correlation was found within

Table 3 Echocardiographic data

\begin{tabular}{|c|c|c|c|c|}
\hline \multirow[b]{2}{*}{ Left ventricular end diastolic diameter $(\mathrm{mm})$} & $H C M(n=17)$ & $H T(n=12)$ & \multicolumn{2}{|c|}{$N(n=10)$} \\
\hline & (11) & (6) & 48 & (5) \\
\hline Left atrial end systolic diameter (mm) & $(9) \dagger$ & (5) & 36 & (5) \\
\hline Interventricular septal thickness (mm) & $(6)^{\star}$ & $(2)^{\star}$ & 9 & $(2)^{\star}$ \\
\hline Posterior wall thickness (mm) & $(4) \dagger$ & (2) & 9 & (2) \\
\hline Left ventricular mass index $\left(\mathrm{g} / \mathrm{m}^{2}\right)$ & $(63)^{\star}$ & 137 & 81 & (23) \\
\hline Wall thickness index (mm) & (17) & 127 & 76 & $(9)^{\star \star}$ \\
\hline Asymmetry index & $0.31(0.10)^{\star \star}$ & $0.10(0.05)$ & 0.1 & $(0.01)$ \\
\hline Left ventricular ejection fraction (\%) & $(7) \dagger$ & (11) & 65 & (3) \\
\hline Mean left ventricular outflow tract gradient (mm Hg) & $(23)^{\star}$ & (1) & 4 & (1) \\
\hline Isovolumetric relaxation time (ms) & (26) & (16) & 70 & $(10)^{\star}$ \\
\hline $\mathrm{E} / \mathrm{A}$ & $0.98(0.35)$ & $0.88(0.41) \dagger$ & 1.3 & $(0.39)$ \\
\hline Deceleration time of early diastolic left ventricular filling (ms) & $(67) \dagger$ & 211 & 162 & (17) \\
\hline Asynchrony index & $0.13(0.07) \dagger$ & $0.08(0.04)$ & 0.0 & $(0.03)$ \\
\hline
\end{tabular}

Values are mean (SD).

tp $<0.05 v$ normal group; ${ }^{\star} \mathrm{p}<0.05 v$ the other two groups; ${ }^{\star \star} \mathrm{p}<0.001 v$ the other two groups. 
Table 4 Correlations of the extent and distribution of left ventricular hypertrophy with Doppler indices of regional and global left ventricular relaxation

\begin{tabular}{|c|c|c|c|c|}
\hline & All groups $(n=39)$ & $H C M(n=17)$ & $H T(n=12)$ & $N(n=10)$ \\
\hline \multicolumn{5}{|l|}{ Regional wall thickness } \\
\hline rIVRT & $r=0.55, \mathrm{p}<0.0001$ & $r=0.47, \mathrm{p}<0.0001$ & $r=0.21, \mathrm{p}<0.05$ & NS \\
\hline $\mathrm{rE} / \mathrm{A}$ & $r=-0.44, \mathrm{p}<0.0001$ & $r=-0.20, \mathrm{p}<0.05$ & $r=-0.30, \mathrm{p}=0.003$ & NS \\
\hline \multicolumn{5}{|c|}{ Left ventricular asymmetry index } \\
\hline Coeff var $\mathrm{rE} / \mathrm{A}$ & NS & NS & NS & NS \\
\hline Asynchrony index & $r=0.49, \mathrm{p}=0.002$ & NS & NS & NS \\
\hline \multicolumn{5}{|c|}{ Left ventricular wall thickness index } \\
\hline $\mathrm{E} / \mathrm{A}$ & $r=-0.33, \mathrm{p}<0.05$ & NS & NS & NS \\
\hline IVRT & $r=0.39, \mathrm{p}=0.01$ & NS & NS & NS \\
\hline E-DT & $r=0.42, \mathrm{p}=0.007$ & NS & $r=0.67, \mathrm{p}=0.02$ & NS \\
\hline \multicolumn{5}{|c|}{ Left ventricular asymmetry index } \\
\hline $\mathrm{E} / \mathrm{A}$ & NS & NS & NS & NS \\
\hline IVRT & NS & NS & NS & NS \\
\hline E-DT & $r=0.50, \mathrm{p}=0.001$ & NS & NS & NS \\
\hline
\end{tabular}

Coeff var, coefficient of variation; E/A, early to late diastolic transmitral flow velocity ratio; E-DT, deceleration time of early transmitral filling; HCM, hypertrophic cardiomyopathy; HT, hypertensive heart disease; IVRT, isovolumetric relaxation time; N, normal controls; rE/A, regional early to late peak diastolic myocardial velocity ratio; rIVRT, regional isovolumetric relaxation time.

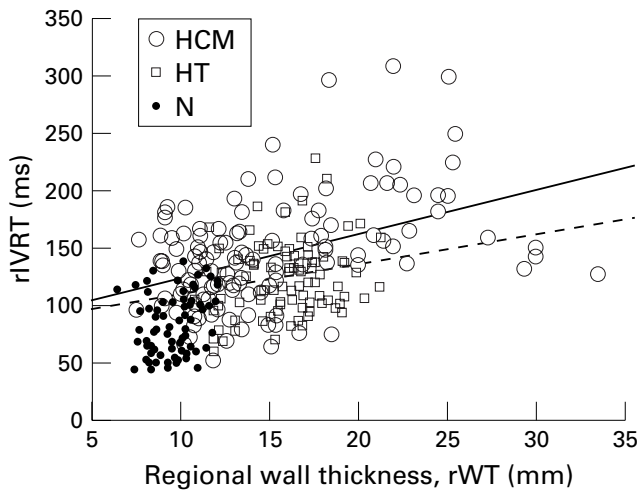

Figure 2 Relation between regional wall thickness ( $r W T$, $x$ axis) and regional isovolumetric relaxation time (rIVRT, $y$ axis). There was a positive correlation between these two variables in both hypertrophic cardiomyopathy (HCM, empty circles, continuous regression line: $y=85+4.0 x ; r=$ $0.47, p<0.0001)$ and hypertensive heart disease (HT, rectangles, dashed regression line: $y=82+2.8 x ; r=0.21$, $p<0.0001)$.

individual study groups. However, a majority of patients with hypertrophic cardiomyopathy (9/17) had an abnormal asynchrony index (that is, values exceeding the upper 95 th centile of the values of the normal group, $\mathrm{p}<0.05$, fig 3 ) compared with the other groups (1/12 patients with hypertensive heart disease, 1/10 normal subjects). Patients with hypertrophic cardiomyopathy with a normal asynchrony index did

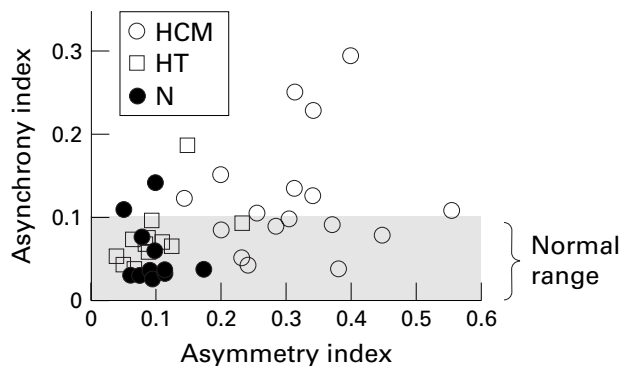

Figure 3 There was a significant correlation between left ventricular asymmetry $(x$ axis) and the asynchrony index (y axis) $(y=0.05+0.23 x ; r=0.49, p=0.02)$, but not for the individual groups. Patients with abnormal asynchrony index did not differ from patients with normal asynchrony index in terms of left ventricular asymmetry. HCM. Hypertrophic cardiomyopathy; HT, hypertensive heart disease; $N$, normal. not differ in terms of left ventricular asymmetry from patients with an abnormal asynchrony index.

LEFT VENTRICULAR HYPERTROPHY AND DOPPLER INDICES OF GLOBAL LEFT VENTRICULAR RELAXATION

In none of the groups was there a correlation between left ventricular wall thickness index (that is, the extent of hypertrophy) and the E/A ratio of transmitral filling or the isovolumetric relaxation time (table 4 ). The left ventricular wall thickness index was significantly related to the deceleration time of early transmitral filling (E-DT) in the hypertensive heart disease group but not in the other groups. There was no correlation between asymmetry index and E/A ratio, isovolumetric relaxation time, or E-DT within the different groups (table 4).

DOPPLER INDICES OF REGIONAL AND GLOBAL LEFT VENTRICULAR RELAXATION

Asynchrony index was highest in the cardiomyopathy group, lowest in the normal group, and intermediate in the hypertensive heart disease group. The difference between the cardiomyopathy group and the normal group was significant, but not the other comparisons. Mean rIVRT was linearly related to isovolumetric relaxation time, and mean $\mathrm{rE} / \mathrm{A}$ was linearly related to E/A in each group (table 5).

A strong positive overall correlation was found between asynchrony index and E-DT (fig 4). The correlation was significant for the cardiomyopathy group as well as for the hypertensive group, but not for the normal controls (table 5). There was a significant overall inverse correlation between the asynchrony index and both the E/A ratio of transmitral inflow and the isovolumetric relaxation time (table 5), but not within the individual groups. In the cardiomyopathy group, however, the nine patients with an abnormal asynchrony index had a prolonged isovolumetric relaxation time compared with the eight patients with a normal asynchrony index (108 (25) ms v 83 (23) ms, $\mathrm{p}<0.05)$. There were no differences in terms of age, left ventricular outflow tract gradient, left ventricular wall thickness, or left ventricular wall thickness index between these two sub- 
Table 5 Correlations between Doppler indices of regional and global left ventricular relaxation

\begin{tabular}{lllll}
\hline & All groups $(n=39)$ & $H C M(n=17)$ & $H T(n=12)$ & $N(n=10)$ \\
\hline $\begin{array}{l}\text { Mean } r \text { IVRT } \\
\text { IVRT }\end{array}$ & $r=0.68, \mathrm{p}<0.0001$ & $r=0.51, \mathrm{p}=0.04$ & $r=0.63, \mathrm{p}=0.03$ & $r=0.77, \mathrm{p}<0.01$ \\
$\begin{array}{l}\text { Mean } r \text { E/A } \\
\text { E/A }\end{array}$ & $r=0.73, \mathrm{p}<0.0001$ & $r=0.74, \mathrm{p}<0.001$ & $r=0.64, \mathrm{p}=0.03$ & $r=0.70, \mathrm{p}=0.02$ \\
$\begin{array}{ll}\text { Asynchrony index } \\
\text { E/A }\end{array}$ & $r=-0.39, \mathrm{p}=0.01$ & $\mathrm{NS}$ & $\mathrm{NS}$ & $\mathrm{NS}$ \\
IVRT & $r=0.51, \mathrm{p}=0.001$ & $\mathrm{NS}$ & $\mathrm{NS}$ & $\mathrm{NS}$ \\
E-DT & $r=0.69, \mathrm{p}<0.0001$ & $r=0.56, \mathrm{p}=0.02$ & $r=0.59, \mathrm{p}<0.05$ & NS \\
\hline
\end{tabular}

See table 4 for abbreviations.

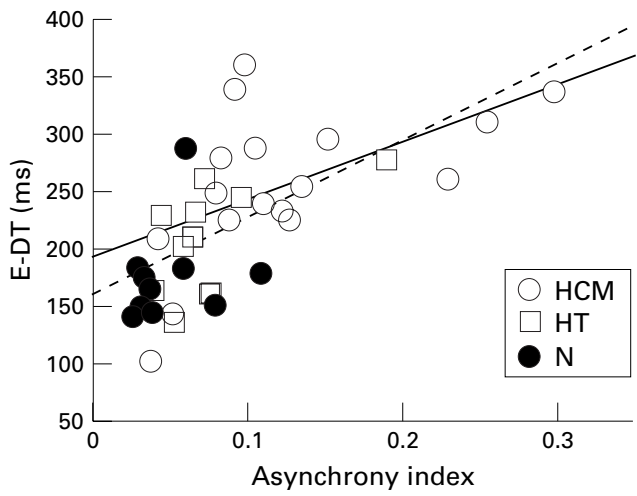

Figure 4 Relation between asynchrony index ( $x$ axis) and deceleration time of early transmitral filling ( $E-D T$, y axis). The correlations were significant for both hypertrophic cardiomyopathy (HCM, empty circles, uninterrupted regression line: $y=192+509 x ; r=0.56, p=0.02)$ and hypertensive heart disease (HT, rectangles, dashed regression line; $y=158+680 x ; r=0.59, p<0.05)$.

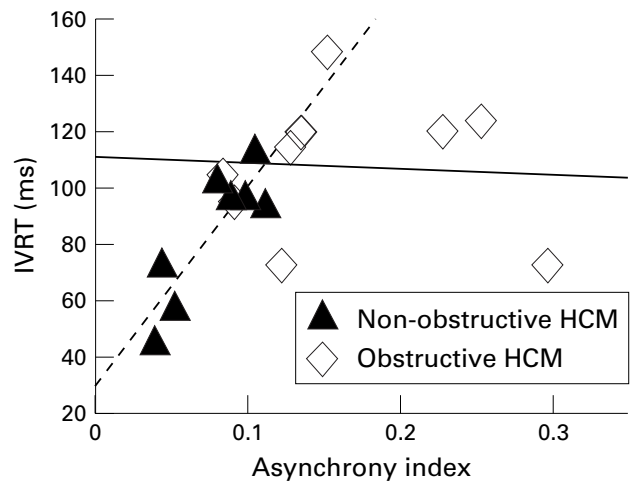

Figure 5 Relation between asynchrony index ( $x$ axis) and isovolumetric relaxation time (IVRT, $y$ axis) in patients with hypertrophic cardiomyopathy (HCM) who did not have left ventricular outflow tract obstruction (triangles, dashed regression line) and those who did (rectangles, uninterrupted regression line). In patients with outflow tract obstruction, no correlation was found; in those with no outflow tract obstruction, IVRT was linearly related to the asynchrony index $(y=29+716 x ; r=0.87 ; p=0.005)$.

populations in the cardiomyopathy group. The correlation between asynchrony index and isovolumetric relaxation time was excellent in the cardiomyopathy patients who did not have left ventricular outflow tract obstruction $(r=0.87$, $\mathrm{p}=0.005)$, whereas in those with significant obstruction (mean gradient $=10 \mathrm{~mm} \mathrm{Hg}$ ), no relation was found (fig 5).

\section{Discussion}

Non-invasive determination of regional left ventricular myocardial wall thickness together with appropriate local Doppler tissue imaging has made it possible for the first time to study the relation between regional morphological abnormalities in hypertrophied ventricles and global relaxation abnormalities. In this study we found a close association between the regional extent of hypertrophy and abnormalities of relaxation in both hypertrophic cardiomyopathy and hypertensive heart disease. Over the entire range from normal to hypertrophied hearts, the degree of asymmetry in regional wall thickness was linked to the temporal dispersion of regional cardiac cycle events (asynchrony), while the asynchrony was itself related to impaired global left ventricular filling in both hypertrophic cardiomyopathy and hypertensive heart disease.

\section{LEFT VENTRICULAR HYPERTROPHY AND RELAXATION}

There have been many studies showing that structural heart diseases with left ventricular hypertrophy is associated with left ventricular relaxation abnormalities. ${ }^{2} 3031$ In hypertrophic cardiomyopathy, however, it has been found that the Doppler pattern of left ventricular filling is largely independent of the extent of left ventricular hypertrophy. ${ }^{30}$ Focusing on the entire ventricle, our present study confirms these findings, showing an independence of the conventional Doppler diastolic relaxation indices from the extent of left ventricular hypertrophy (that is, left ventricular wall thickness index) in hypertrophic cardiomyopathy. Regional analysis using Doppler tissue imaging, however, showed that increasing regional wall thickness was associated with increasing regional relaxation disturbance in both hypertrophic cardiomyopathy and hypertensive heart disease. The similarity of these associations between the two groups allows one to speculate that the functional result of increased wall thickness on myocardial relaxation does not differ substantially between primary and secondary left ventricular hypertrophy. Furthermore, abnormal relaxation could also be found in macroscopically normal wall segments among patients with hypertrophic cardiomyopathy and less frequently in those with hypertensive heart disease (fig 2), showing that overt myocardial hypertrophy may not be necessary for the development of diastolic dysfunction. Factors other than local wall thickness that may influence relaxation, such as regional intraventricular pressure differences and varying ventricular shapes, were not measured directly in this study. This may explain a considerable amount of the variability in the association between regional wall thickness and regional isovolumetric relaxation (fig 2). In addition, measurement variability of regional relaxation 
indices may have been caused by the translational motion of the heart.

Another question we addressed was whether unequal distribution of left ventricular hypertrophy (asymmetry) is associated with abnormal regional and global left ventricular relaxation. This has been suggested by earlier investigations, ${ }^{92-34}$ but cannot be directly confirmed by our present study. However, asymmetry was associated with asynchrony in the entire study population, but not within the individual study groups. Thus, asymmetrical left ventricular hypertrophy appears to be a predisposing - that is, necessary but insufficient on its own-condition for the development of asynchrony (fig 3).

COMPARISON BETWEEN REGIONAL AND GLOBAL DIASTOLIC EVENTS

By investigating the relation between the structural features of left ventricular hypertrophy (extent and asymmetry) and left ventricular filling, which as the purpose of this study, we implicitly sought also to evaluate how regional relaxation is related to global relaxation in the left ventricle. The high temporal resolution of Doppler tissue imaging makes it possible to determine accurately specific events of the cardiac cycle in different myocardial regions. The end of ventricular contraction is easily recognisable and was therefore chosen as a hallmark event during the phase of myocardial relaxation. The temporal dispersion of this event throughout the different regions was presumed to reflect the asynchrony of contractionrelaxation coupling. A similar approach has recently been proposed by Pai and Gill. ${ }^{21}$ As expected, highly asynchronous ventricular long axis function was seen in the patients with hypertrophic cardiomyopathy. Left ventricular long axis motion is the effect of contraction of longitudinally oriented fibres, producing basoapical shortening of the ventricle.

To determine the functional relevance of long axis asynchrony for global left ventricular relaxation, the asynchrony index was compared with Doppler indices of left ventricular filling. On the basis of analysis of all three study groups, asynchrony was associated with a decrease in transmitral E/A ratio, protracted early filling, and prolonged isovolumetric relaxation time. The value of conventional Doppler indices in assessing relaxation is, however, controversial. ${ }^{35}$ An important factor influencing these variables - age-was corrected for by matching the study group individuals. For the cardiomyopathy group in particular, there was a significant correlation between the asynchrony index and the deceleration time of early filling - that is, a link between regional and global early diastolic events could be shown. In the absence of left ventricular outflow tract obstruction, isovolumetric relaxation time was also closely related to asynchrony. In the cardiomyopathy patients with left ventricular outflow tract obstruction, and also in the patients with hypertensive heart disease, this correlation was lacking. This may reflect the combined influence of the haemodynamic conditions within the left ventricular outflow tract and aortic pressure on the onset of aortic valve closure.

STUDY LIMITATIONS

Doppler tissue imaging variables for each region under investigation were assessed from different cardiac cycles. Asynchrony could not therefore be calculated from a single heart beat and so could have been affected by changes in heart rate. Within the different groups, however, heart rate did not alter significantly during the study. Furthermore, the time interval used to measure asynchrony included only the systolic phase of the cardiac cycle, which is only moderately affected by changes in heart rate.

Coronary heart disease can account for asynchronous heart action. In our patients, coronary angiography was not performed as a part of the study protocol. We cannot therefore entirely exclude the presence of coronary heart disease, although none of the patients suffered from angina pectoris.

Patients with hypertrophic cardiomyopathy were significantly more often on calcium antagonists (verapamil) than patients in the other groups. Verapamil has a known beneficial effect on left ventricular relaxation and asynchrony. ${ }^{36}$ The profile of the cardiomyopathy group may therefore not entirely reflect the natural history of the disease. However, it is likely that the findings would have been even more pronounced in the absence of calcium antagonists.

\section{CONCLUSIONS}

Non-invasive ultrasonographic examination of the left ventricle shows that in hypertrophic cardiomyopathy as well as in hypertensive heart disease the local extent of left ventricular hypertrophy is associated with regional left ventricular relaxation abnormalities. Asymmetrical distribution of left ventricular hypertrophy is indirectly related to global left ventricular early filling abnormalities through regional asynchrony of left ventricular relaxation.

1 Grossman W, Jones D, McLaurin LP. Wall stress and patterns of hypertrophy in the human left ventricle. $f$ Clin Invest 1975;56:56-64.

2 Goodwin JF. Congestive and hypertrophic cardiomyopathies. A decade of study. Lancet 1970;i:732-9.

3 Shepherd RF, Zachariah PK, Shub C. Hypertension and left ventricular diastolic function. Mayo Clin Proc 1989;64: 1521-32.

4 Skelton CL, Sonnenblick EH. Heterogeneity of contractile function in cardiac hypertrophy. Circ Res 1974;35(suppl II): $83-96$

5 Yelamarty RV, Moore RL, Yu FT, et al. Relaxation abnormalities in single cardiac myocytes from renovascular hypertensive rats. Am $\mathcal{F}$ Physiol 1992;262:C980-90

6 Hess OM, Schneider J, Koch R, et al. Diastolic function and myocardial structure in patients with myocardial hypertrophy. Special reference to normalized viscoelastic data. Circulation 1981;63:360-71.

7 Shapiro LM, McKenna WJ. Left ventricular hypertrophy. Relation of structure to diastolic function in hypertension. Br Heart f 1 984;51:637-42.

8 Douglas PS, Tallant B. Hypertrophy, fibrosis and diastolic dysfunction in early canine experimental hypertension. $\mathcal{F}$ Am Coll Cardiol 1991;17:530-6.

9 Seiler C, Jenni R, Krayenbuehl HP. Intraventricular blood flow during isovolumetric relaxation and diastole in hypertrophic cardiomyopathy. $\mathcal{f}$ Am Soc Echocardiogr 1991;4: 247-57.

10 Schneider J, Hess OM, Krayenbuhl HP. Muscle fiber hypertrophy and myocardial function of the left ventricle in patients with chronic volume stress and cardiomyopathy [in German]. Schweiz Med Wochenschr 1979;109:1651-2. 
11 Villari B, Campbell SE, Hess OM, et al. Influence of collagen network on left ventricular systolic and diastolic function in a

12 Brutsaert DL, Sys SU. Relaxation and diastole of the heart. Physiol Rev 1989;69:1228-315.

13 Gillebert TC, Lew WY. Nonuniformity and volume loading independently influence isovolumic relaxation rates. $A m \mathscr{F}$ Physiol 1989;257:H1927-35.

14 Abe $\mathrm{H}$, Tomotsune K. Asynchronous relaxation of the ischemic left ventricle. Fpn Circ f 1982;46:103-12.

15 Lew WY, Rasmussen CM. Influence of nonuniformity on rate of left ventricular pressure fall in the dog. Am $\mathcal{F}$ Physiol 1989;256: H222-32

16 Betocchi S, Hess OM, Losi MA, et al. Regional left ventricular mechanics in hypertrophic cardiomyopathy. Circulation 1993;88:2206-14

17 Sohn DW, Chai IH, Lee DJ, et al. Assessment of mitral annulus velocity by Doppler tissue imaging in the
evaluation of left ventricular diastolic function. $7 \mathrm{Am}$ Coll Cardiol 1997;30:474-80.

18 Sutherland GR, Stewart MJ, Groundstroem KW, et al. Color Doppler myocardial imaging: a new technique for the assessment of myocardial function. $\mathcal{F} \mathrm{Am}$ Soc Echocardiogr 1994;7:441-58

19 Nagueh SF, Middleton KJ, Kopelen HA, et al. Doppler tissue imaging: a noninvasive technique for evaluation of left ventricular relaxation and estimation of filling pressures. $\mathcal{F}$ Am Coll Cardiol 1997;30:1527-33.

20 Seiler C, Heule K, de Marchi SF. Accuracy and preloaddependence of diastolic mitral annular velocity parameters for the assessment of "pseudonormal" LV relaxation [abstract]. Eur Heart f 1999;20(suppl):148.

21 21. Pai RG, Gill KS. Amplitudes, durations, and timings of apically directed left ventricular myocardial velocities: II Systolic and diastolic asynchrony in patients with left ventricular hypertrophy. $\mathcal{F}$ Am Soc Echocardiogr 1998;11:11218 .

22 Pai RG, Gill KS. Amplitudes, durations, and timings of apically directed left ventricular myocardial velocities. I. Their normal pattern and coupling to ventricular filling and ejection. $\mathcal{F}$ Am Soc Echocardiogr 1998;11:105-11.

23 Devereux RB, Lutas EM, Casale PN, et al. Standardization of $\mathrm{M}$-mode echocardiographic left ventricular anatomic measurements. F Am Coll Cardiol 1984;4:1222-30.
24 Sahn DJ, De Maria A, Kisslo J, et al. Recommendations regarding quantitation in M-mode echocardiography: results of a survey of echocardiographic measurements. Circulation 1978;58:1072-83.

25 Troy BL, Pombo J, Rackley CE. Measurement of left ventricular wall thickness and mass by echocardiography. Circulation 1972;45:602-11.

26 Spirito P, Maron BJ, Bonow RO, et al. Occurrence and significance of progressive left ventricular wall thinning and relative cavity dilatation in hypertrophic cardiomyopathy. Am 7 Cardiol 1987;60:123-9.

27 Posma JL, Blanksma PK, van der Wall EE, et al. Assessment of quantitative hypertrophy scores in hypertrophic cardiomyopathy: magnetic resonance imaging versus echocardiography. Am Heart f 1996;132:1020-7.

28 McDicken WN, Sutherland GR, Moran CM, et al. Colour McDicken WN, Sutherland GR, Moran CM, et al. Colour
Doppler velocity imaging of the myocardium. Ultrasound Med Biol 1992;18:651-4

29 Miyatake K, Yamagishi M, Tanaka N, et al. New method for evaluating left ventricular wall motion by color-coded tissue Doppler imaging: in vitro and in vivo studies. $\mathcal{f} \mathrm{Am}$ Coll Cardiol 1995;25:717-24.

30 Spirito P, Maron BJ. Relation between extent of left ventricular hypertrophy and diastolic filling abnormalities in hypertrophic cardiomyopathy. 7 Am Coll Cardiol 1990;15:808-13.

31 Hirota Y. A clinical study of left ventricular relaxation. Circulation 1980;62:756-63.

32 Gibson DG, Sanderson JE, Traill TA, et al. Regional left ventricular wall movement in hypertrophic cardiomyopathy. Br Heart f 1978;40:1327-33.

33 Sanderson JE, Gibson DG, Brown DJ, et al. Left ventricular filling in hypertrophic cardiomyopathy. An angiographic study. Br Heart $\mathcal{F} 1977 ; 39: 661-70$.

34 Hanrath P, Mathey DG, Siegert R, et al. Left ventricular relaxation and filling pattern in different forms of left ventricular hypertrophy: an echocardiographic study. Am $\mathcal{f}$ Cardiol 1980;45:15-23.

35 Hatle L. Doppler echocardiographic evaluation of diastolic function in hypertensive cardiomyopathies. Eur Heart 7 1993;14:88-94.

36 Hess OM, Grimm J, Krayenbuehl HP. Diastolic function in hypertrophic cardiomyopathy: effects of propranolol and verapamil on diastolic stiffness. Eur Heart $\mathcal{F} 1983 ; 4: 47-56$. 